\title{
Effects of Plasma Parameters on Viscosity
}

\author{
Hossein Dahi, Joseph N. Talmadge, Member, IEEE, and J. Leon Shohet, Fellow, IEEE
}

\begin{abstract}
A numerical code is developed to study the effect of ion density and temperature (as well as ion pressure and temperature gradients) on the amplitude of the local maxima of the poloidal viscosity with respect to the poloidal Mach number using the model developed by Shaing [1]. The sum of the poloidal viscosity and ion-neutral collisions is determined from the dependence of the plasma radial current on the radial electric field in a biased electrode experiment. The experimental results in the interchangeable module stellarator agree qualitatively with the predictions of the numerical calculation regarding the effects of variation of ion density and temperature on viscosity.
\end{abstract}

Index Terms - Biased electrode experiments, L-H transition, stellarators, viscosity.

\section{INTRODUCTION}

O $\mathrm{NE}$ of the central challenges of the fusion program is improving the confinement time in toroidal magnetic devices. Improvement of the energy confinement through L-H transition was first discovered in axially symmetric divertor experiment (ASDEX) [2]. A jump in the radial electric field and formation of a large shear in the electric field at the plasma edge are among the characteristics of L-H transition [3]. One model to explain the jump in the radial electric field is based on the nonlinearity of the parallel viscosity at large flow speeds [4].

Biased electrode experiments in tokamaks, [5]-[7] have shown that there is a sudden jump in the radial electric field during the L-H transition about a poloidal Mach number $\left(M_{p}=-c E_{r} / B_{p} v_{t}\right.$ where $c$ is the speed of light, $E_{r}$ is the radial electric field, $B_{p}$ is poloidal magnetic field, and $v_{t}$ is the thermal velocity of ions) of unity. These experiments indicated that the viscosity in tokamaks is nonlinear. Spontaneous L-H transitions have also been reported in the Wendelstein VIIAS [8], compact helical system (CHS) [9], and H-1 [10] stellarators. The experimental results from the interchangeable module stellarator (IMS), presented previously [11], indicate that viscosity in a stellarator is nonlinear too.

According to Shaing's model [1] the peak in the poloidal viscosity at a poloidal Mach number close to one in tokamaks occurs because of the purely toroidal modulation of the magnetic field. In addition to the peak in viscosity due to

Manuscript received December 23, 1997; revised August 4, 1998. This work supported by the U.S. Department of Energy under Grant DE-FG0293ER54222.

H. Dahi was with the Torsatron-Stellarator Laboratory, Department of Electrical and Computer Engineering, University of Wisconsin-Madison, Madison, WI 53706 USA. He is now with the Department of Applied Physics and Applied Mathematics, Columbia University, New York, NY 10027 USA (e-mail: dahi@columbia.edu).

J. N. Talmadge and J. L. Shohet are with the Torsatron-Stellarator Laboratory, Department of Electrical and Computer Engineering, University of Wisconsin-Madison, Madison, WI 53706 USA.

Publisher Item Identifier S 0093-3813(98)08836-5. toroidal curvature, stellarators have additional local maxima much greater than one because of the helical modulation of the field.

In this paper, the effects of plasma parameters on the amplitude of viscosity at different poloidal Mach numbers in a tokamak and a stellarator geometry are studied. Increase in the amplitude of a local maximum in viscosity may provide a necessary condition for $\mathrm{L}-\mathrm{H}$ transition to occur in a given magnetic confinement device. It is shown that the variation in plasma collisionality (through varying ion density and temperature) may alter the amplitude of the local peak in the viscosity. The ion pressure and temperature gradient at the plasma edge may break down the odd symmetry of the viscosity with respect to poloidal Mach number and increase the amplitude of the local maxima in viscosity for negative Mach numbers. The results of the biasing experiments in the IMS [12] confirm some of the predictions of the numerical calculations.

The outline of this paper is as follows: Section II reviews the theoretical background and Shaing's expressions for damping due to viscosity and ion-neutral collisions in a tokamak and in a stellarator geometry. The results of the numerical calculations are presented in Section III. Section IV discusses the IMS device and the diagnostics used in the experiments. The experimental results obtained by inducing plasma flows with a triangular voltage signal are presented in Section V. A summary and conclusions are presented in Section VI.

\section{BACKGROUND}

In steady-state, the poloidal momentum balance equation in Hamada coordinate system [13] can be written as:

$$
\frac{B^{\theta} B^{\zeta}}{c}\langle\boldsymbol{J} \cdot \nabla V\rangle=-\left\langle\boldsymbol{B}_{\boldsymbol{p}} \cdot \nabla \cdot \overleftrightarrow{\pi}\right\rangle-v_{\boldsymbol{i}^{-} n} N M\left\langle\boldsymbol{B}_{\boldsymbol{p}} \cdot V\right\rangle
$$

where $B_{p}$ is the poloidal magnetic field, $B^{\theta}$ and $B^{\zeta}$ are poloidal and toroidal contravariant components of the magnetic field, $c$ is the speed of light, $\boldsymbol{J}$ is plasma current density, $V$ is the volume, $\stackrel{\leftrightarrow}{\pi}$ is viscous stress tensor, $v_{i^{-} n}$ is the ionneutral collision frequency, $N$ and $M$ are ion density and mass respectively, and $V$ is the mass flow vector.

The left side of (1) is the radial current flowing in the plasma. Normally in steady state this radial current is zero. However, when a probe inserted into the plasma draws a radial current, the rate of change of the electric field is proportional to the total probe current plus the plasma current. In steady state the total current, i.e., the sum of the plasma plus probe current, vanishes. This forces the plasma radial current in (1) to be nonzero [14]. 
The terms on the right side of (1) are the damping terms due to the viscosity and ion-neutral collisions. The total damping is given by

$$
\begin{aligned}
& \frac{\langle\boldsymbol{B} \boldsymbol{p} \cdot \nabla \cdot \overleftrightarrow{\boldsymbol{\pi}}\rangle}{N M v_{t}^{2} B^{\theta}}+v_{i-n} \frac{\left\langle\boldsymbol{B}_{\boldsymbol{p}} \cdot \boldsymbol{V}\right\rangle}{v_{t}^{2} B^{\theta}} \\
& =\sqrt{\frac{\pi}{4}} \sum_{\substack{m, n=-\infty \\
m \neq 0}}^{\infty} \varepsilon_{m n}^{2} m^{2}\left[I_{m n}\left(M_{p}-V_{p p}\right)-L_{m n} V_{p t}\right] \\
& \quad+v_{i-n} \frac{R_{0} \varepsilon^{2}}{q v_{t}}\left(1+2 q^{2}\right)\left(M_{p}-V_{p p}\right)
\end{aligned}
$$

where

$$
\begin{aligned}
M_{p}= & -\frac{c E_{r}}{B_{p} v_{t}}, \quad V_{p p}=-\frac{c}{e N B_{p} v_{t}} \frac{d p}{d r} \\
V_{p t}= & -\frac{c}{e B_{p} v_{t}} \frac{d T}{d r} \\
\left\{\begin{array}{c}
I_{m n} \\
L_{m n}
\end{array}\right\}= & \frac{1}{\pi(m-n q)} \int_{0}^{\infty} d x x^{2} \exp (-x) \\
& \cdot\left\{\begin{array}{c}
1 \\
x-2.5
\end{array}\right\} \int_{-1}^{1} d y\left(1-3 y^{2}\right)^{2} \frac{c_{1}}{\left(y+c_{2}\right)^{2}+c_{1}^{2}}
\end{aligned}
$$

and

$$
c_{1}=\frac{q R_{0} v_{k}}{(m-n q) \sqrt{x v_{t}}}, \quad c_{2}=\frac{m M_{p}}{(m-n q) \sqrt{x}}
$$

$p$ and $T$ are ion pressure and temperature, $R_{0}$ is the major radius, $r$ is the minor radius, $v_{k}$ is the Krook collision frequency, $M_{p}, V_{p p}$, and $V_{p t}$ are normalized $\boldsymbol{E} \times B$ flow (poloidal Mach number), pressure gradient, and ion temperature gradient, respectively, $m$ and $n$ are the poloidal and toroidal mode numbers, $\varepsilon_{m n}$ is the mode amplitude, $v_{t}$ is the thermal velocity, $y=v_{\|} / v, x=v^{2} / v_{t}^{2}, v$ is the velocity of a particle, $v_{\|}$is the parallel component of the velocity of a particle, and $q$ is the safety factor on a magnetic flux surface.

According to (2) damping due to ion-neutral collisions depends linearly on $M_{p}$ and, hence, is dominant at higher flow speeds. Viscous damping, on the other hand, is dominant at lower flow speeds and diminishes at higher poloidal Mach numbers. Since damping due to viscosity and ion-neutral collisions are both functions of the poloidal Mach number, then according to (1), the dependence of the plasma radial current on the radial electric field characterizes the total damping.

A simple physical picture of the local maxima in the viscosity can be understood based on the resonance term in the denominator of the energy integrals $I_{m n}$ and $L_{m n}, y=-c_{2}$ or when $M_{p}$ is approximately equal to $(m-n q) / m$

$$
(m-n q) v_{\|} B_{p} / B=m U_{\boldsymbol{E} \times \boldsymbol{B}}
$$

where $U_{\boldsymbol{E} \times \boldsymbol{B}}=c E_{r} / B$. For a given helical harmonic described by $\cos (m \theta-n \zeta)$, the term $(m-n q)$ on the left side of (4) is the number of bumps in the magnetic field encountered by moving along the magnetic field line a distance once around poloidally. The term $v_{\|} B_{p} / B$ is the component of the parallel velocity in the poloidal direction. The left side of (4) is proportional then to the transit frequency of a particle in a magnetic ripple. Similarly, the term on the right is proportional to the transit frequency of the $\boldsymbol{E} \times \boldsymbol{B}$ flow. The resonance occurs when the two transit frequencies are equal. For the case of a tokamak, the local maximum due to the toroidal curvature is located at $M_{p} \sim 1(m=1, n=0)$. For a stellarator, the local maximum due to the helical curvature along the magnetic field line is typically located at poloidal Mach numbers much greater than one (at $M_{p} \sim|m-n q| / m$ ).

Equations (2) and (3) also indicate that for the case of $V_{p p}=$ $V_{p t}=0$, viscosity is an odd function of the poloidal Mach number (note that the energy integrals $I_{m n}$ and $L_{m n}$ are even functions of $M_{p}$ ). Inclusion of ion pressure and temperature gradients breaks down this odd symmetry. In (3), the terms $c_{1}$ and $y+c_{2}$ in the energy integrals $I_{m n}$ and $L_{m n}$ represent the collisional and $\boldsymbol{E} \times \boldsymbol{B}$ frequencies, respectively. In the limit of $c_{1} \gg y+c_{2}$, the resonance effect of $y=-c_{2}$ cannot be observed. Physically, this occurs when particles scatter out of the magnetic ripples because of the high collisionality. Note that the ion collisionality increases through a decrease in the ion temperature or an increase in plasma as well as neutral density. The resonance effect can be observed more clearly when $c_{1} \leq y+c_{2}$. The competition between these frequencies also affects the amplitude of the local maximum in viscosity. The collisional term $c_{1}$ is linearly proportional to the ion-ion collision frequency $\left(v_{k} \propto v\right)$. In this paper the normalized collisionality is given by the quantity $\nu_{* i}=(v R q) /\left(v_{t} \varepsilon^{3 / 2}\right)$, where $v$ is the ion-ion collision frequency and $\varepsilon=\varepsilon_{10}$.

\section{NUMERICAL CALCULATIONS}

The only nonlinear terms in (2) are the energy integrals $I_{m n}$ and $L_{m n}$. To investigate the dependence of the energy integrals on $v_{* i}$, the results of the numerical calculation of the energy integrals $I_{10}$ and $L_{10}$ for four different values of $v_{* i}$ are shown in Fig. 1. The solid, dash-dot, dashed, and dotted curves correspond to $v_{* i}=1,10,30$, and 100 , respectively. For $v_{* i}=1$, both of the energy integrals have three local maxima at $M_{p}=0$ and $\sim \pm 2$. The decay of both $I_{10}$ and $L_{10}$ at higher poloidal Mach numbers becomes less rapid as $v_{* i}$ is increased. The amplitude of the local maxima also decreases when $v_{* i}$ is increased.

Fig. 2 shows the effects of ion pressure and temperature gradient $\left(V_{p p}\right.$ and $\left.V_{p t}\right)$ on the normalized viscosity as a function of poloidal Mach number for a tokamak geometry. For all of the curves shown in this figure, the same plasma parameters (i.e., ion density and temperature, major radius, and magnetic geometry) are used for the energy integrals. The dashed curves are for the case where both $V_{p p}$ and $V_{p t}$ are set to zero. Only positive values of $V_{p p}$ and $V_{p t}$ are considered here, since for most of the practical cases in tokamaks, ion pressure and temperature gradient decrease with an increase in the minor radius at plasma edge. Fig. 2(a) shows the effects of variation in pressure gradient on viscosity ( $V_{p t}$ is set to zero). The dash dot and the solid curves correspond to $V_{p p}=1$ and 3, respectively. Fig. 2(b) shows the effect of variation in ion temperature gradient on viscosity ( $V_{p p}$ is set to zero). 


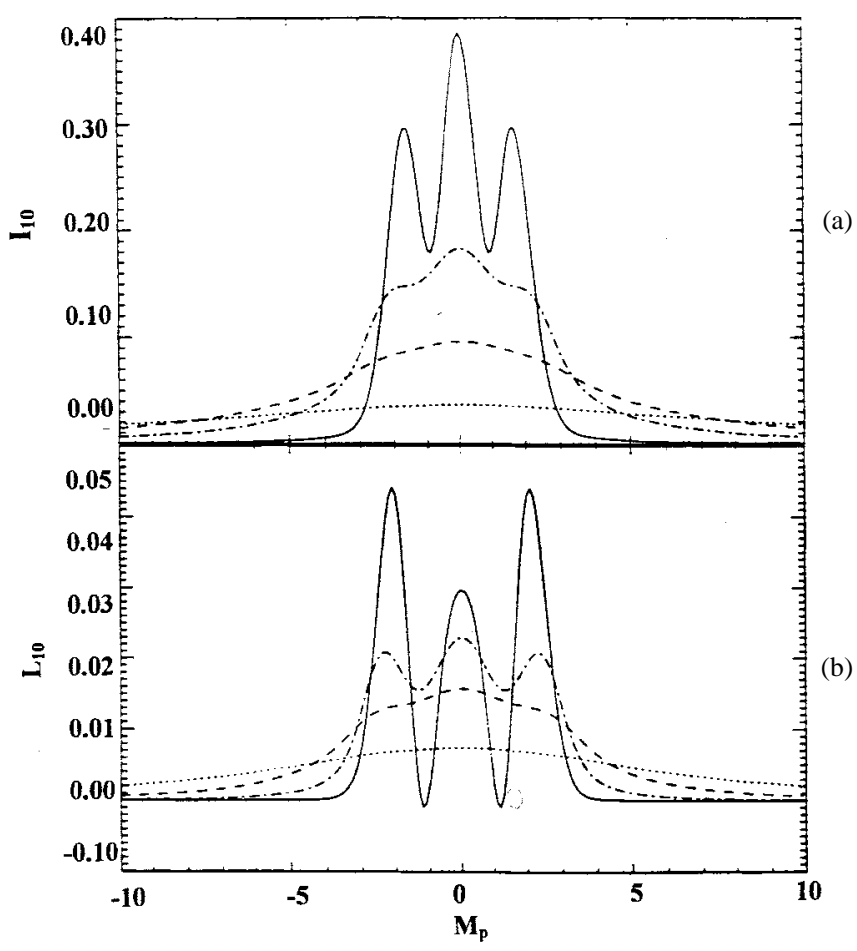

Fig. 1. Energy integrals (a) $I_{10}$ and (b) $L_{10}$ for $\nu_{*} i=1$ (solid), 10 (dash-dot), 30 (dashed), and 100 (dotted).

The dash dot and the solid curves correspond to $V_{p t}=1$ and 3, respectively. Fig. 2(c) shows the effect of variation in both ion temperature and pressure gradient on viscosity. The dash dot and the solid curves correspond to $V_{p t}=V_{p p}=0.5$ and 1.0, respectively. All of these figures indicate that the odd symmetry of viscosity with poloidal Mach number breaks down and the amplitude of viscosity at negative Mach numbers increases as $V_{p t}$ and $V_{p p}$ are increased.

In contrast to the poloidal viscosity calculations for a tokamak, which only has the toroidal curvature term in the magnetic field spectrum, we now consider the case of a modular stellarator, i.e., the IMS (further description of the IMS is given in the next section). Calculations of the viscous and ion-neutral damping in IMS were done based on a Fourier decomposition of the magnetic field in Hamada coordinates. Up to 57 harmonics are included for these calculations.

Fig. 3(a) shows the viscosity curves as a function of poloidal Mach number for major radii $R=40.7,41.3,41.9 \mathrm{~cm}$ (the magnetic axis is located at $R=38.0 \mathrm{~cm}$ ) in IMS for $T_{i}=7$ $\mathrm{eV}$ and $n_{i}=2.5 \times 10^{11} \mathrm{~cm}^{-3}$ (for the sake of simplicity, the pressure and ion temperature gradients are ignored). The poloidal viscosity increases with minor radius because the "bumpiness" of the magnetic field increases toward the plasma edge. There are two distinct local maxima for each curve. The local maximum which is located at $M_{p} \sim 2$ for all of the major radii, is the toroidal peak due to the toroidal curvature term in the magnetic field spectrum $(m=1, n=0)$. The amplitude of this peak increases toward the plasma edge due to the increase in the magnitude of the mode amplitude $\varepsilon_{1,0}$ with the minor radius in IMS $\left(\varepsilon_{1,0}=0.049,0.065\right.$, and 0.082 for $R=40.7,41.3,41.9 \mathrm{~cm}$, respectively). The amplitude

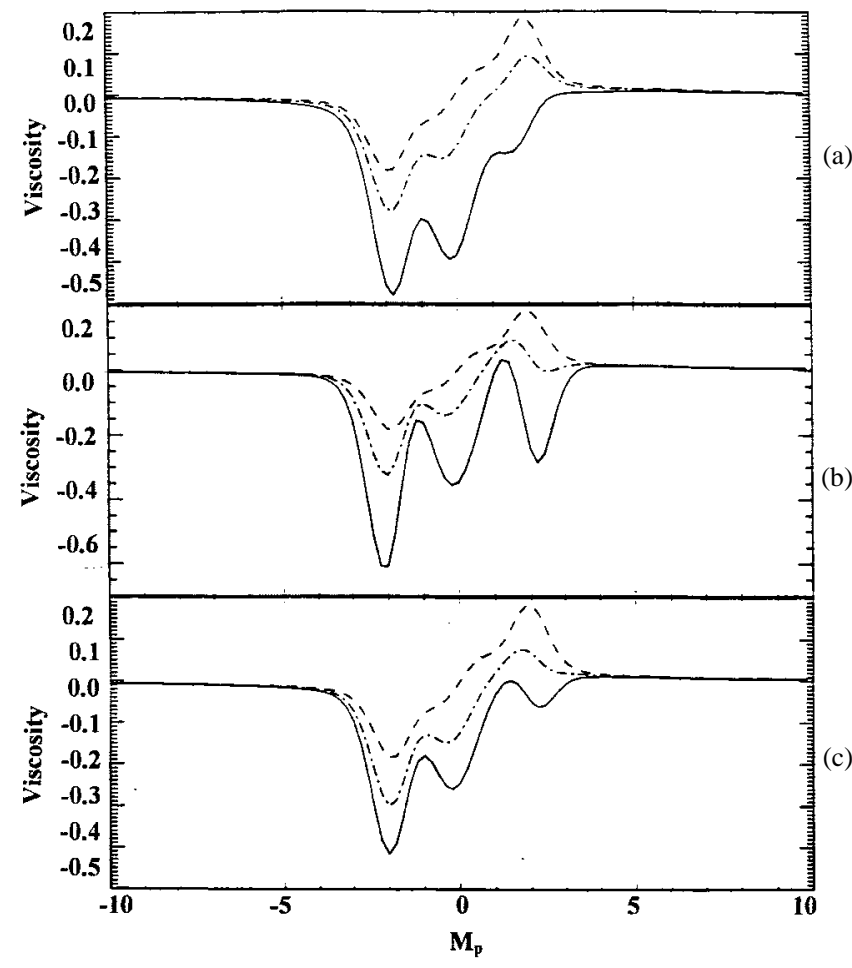

Fig. 2. Viscosity versus poloidal Mach number in a tokamak. (a) $V_{p t}=0$ and $V_{p p}=0$ (dashed), 1 (dash dot), and 3 (solid). (b) $V_{p p}=0$ and $V_{p t}=0$ (dashed), 1 (dash dot), and 3 (solid). (c) $V_{p p}=V_{p t}=0$ (dashed), 0.5 (dash dot), and 1.0 (solid).

of the safety factor $q$ decreases with minor radius in IMS $(q=0.83,0.53$, and 0.36 for $R=40.7,41.3,41.9 \mathrm{~cm}$, respectively). The dominant helical component of the spectrum at all major radii is $m=4, n=7\left(\varepsilon_{4,7}=0.002,0.008\right.$, and 0.0018 for $R=40.7,41.3,41.9 \mathrm{~cm}$, respectively). The location of the other local maximum, which we will refer to as the helical peak is at $M_{p} \sim 13,10,8$ for $R=40.7,41.3$, and $41.9 \mathrm{~cm}$, respectively. The variation of the location of the helical peak $\left(M_{p} \approx|m-n q| / m\right)$ as a function of major radius is a result of the IMS magnetic geometry in which $q$ becomes smaller at the edge. Viscosity vanishes at higher flow velocities $\left(M_{p} \geq 15\right)$ for all major radii.

Fig. 3(b) shows the total damping at $R=41.3, v_{* i}=$ $166, V_{p p}=V_{p t}=1.8$, for $N_{n}=0,18$, and $90 \mu$ torr. The damping due to ion-neutral collisions is proportional to $M_{p}$ and, therefore, the total damping is dominated by viscosity at lower poloidal Mach numbers. At higher poloidal Mach numbers, however, ion-neutral collisions becomes the dominant mechanism for damping of flows. At low neutral densities, e.g., $N_{n}=18 \mu$ torr, both the helical and the toroidal peaks (more clearly for negative poloidal Mach numbers) are present and the nonlinearity of viscosity due to the existence of these peaks does not vanish. For higher neutral density, the helical maximum in viscosity disappears.

Fig. 3(c) shows the effect of variation in $v_{* i}$ on viscosity at $R=41.3 \mathrm{~cm}\left(N_{n}=0\right)$. The amplitude of the toroidal peak decreases and the amplitude of the helical peak increases as $v_{* i}$ is increased. The decrease in the toroidal peak is similar to the behavior of $I_{10}$ in Fig. 1. Varying $v_{* i}$ can provide a 


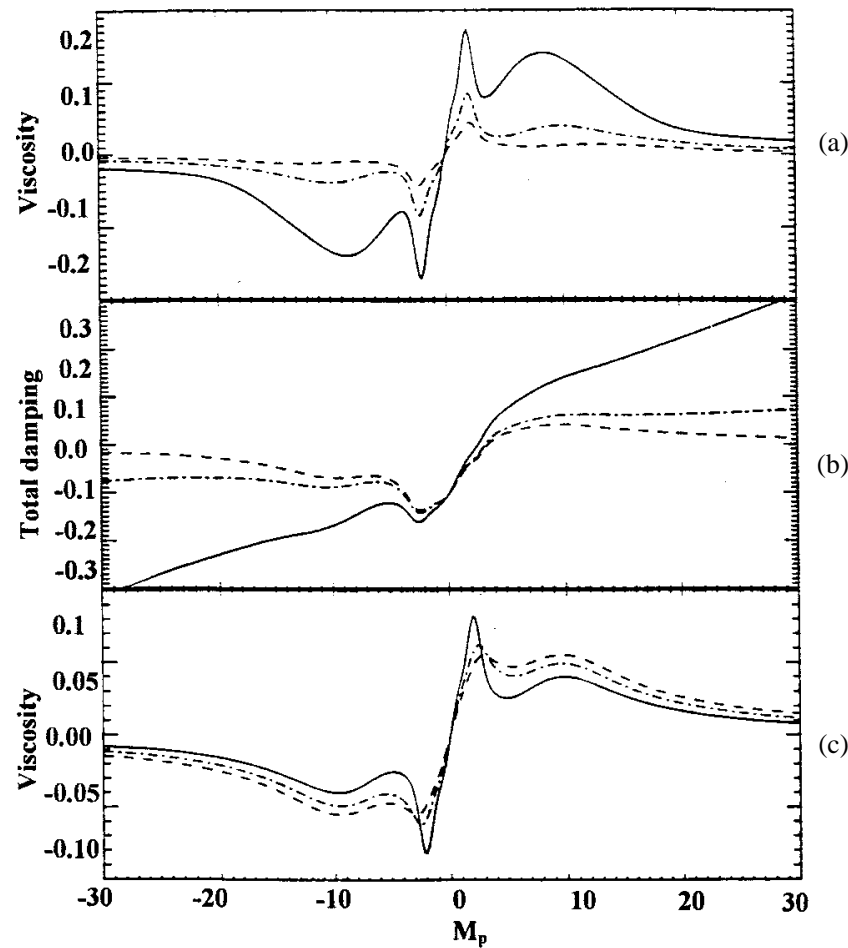

Fig. 3. (a) Viscosity versus $M_{p}$ in the IMS for $R=40.7 \mathrm{~cm}$ (dashed), 41.3 $\mathrm{cm}$ (dash dot), and $41.9 \mathrm{~cm}$ (solid). (b) The total damping versus $M_{p}$ in the IMS for $N_{n}=0$ (dashed), 18 (dash dot), and 90 (solid) $\mu$ torr. (c) Viscosity versus $M_{p}$ in the IMS for $v=35$ (solid), 104 (dash dot), and 166 (dashed)

method to vary the relative magnitude of the two peaks. One important implication of this observation is that higher neutral densities will be required to diminish the helical peak when $v_{* i}$ is increased, or, by increasing $v_{* i}$ the diminished helical peak may reappear. In IMS, the input power can be varied over a wide range $(0.5-6.0 \mathrm{~kW})$, and this can result in production of plasma with different ion densities and temperatures. $v_{* i}$ is more strongly dependent on the ion temperature rather than ion density $\left(v_{* i} \propto n_{i} T_{i}^{-2}\right)$. The effects of the ion pressure and temperature gradient on the viscosity in IMS are very similar to those described in the case of tokamaks.

\section{Diagnostics AND SeTuP}

IMS is a seven field period $l=3$ modular stellarator (where $l$ is the poloidal field period number), with $40 \mathrm{~cm}$ major radius and $\sim 4 \mathrm{~cm}$ average plasma radius. Hydrogen plasmas are produced with an electron cyclotron heating source (0.5-6.0 $\mathrm{kW}$ ) operating at $9.31 \mathrm{GHz}$ and the magnetic field strength $B_{0}=0.31$ Tesla. The neutral density can be varied in the range $N_{n}=18-260 \mu$ torr. Calculations of the neutral density profile indicate a fairly uniform profile since the mean free path for ionization (due to electron impact ionization and dissociation) is much greater than the minor radius. Typical plasma parameters are electron temperatures in the range of 6-20 $\mathrm{eV}$, ion temperatures in the $2-8 \mathrm{eV}$ range, and line average densities of $1-3 \times 10^{11} \mathrm{~cm}^{-3}$. Plasma and floating potentials as well as electron density and temperatures are measured with a Langmuir probe. Line-averaged plasma density is monitored using a $35 \mathrm{GHz}$ microwave interferometer. Ion temperatures

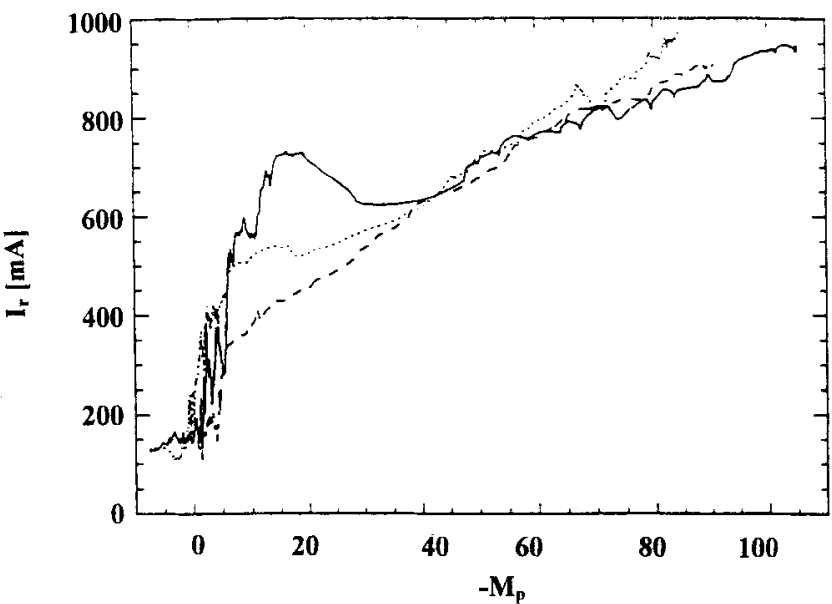

Fig. 4. The radial current versus Mach number, for $N_{n}=18$ (solid), 40 (dotted), and 80 (dashed) $\mu$ torr, at $P_{r f}=2.0 \mathrm{~kW}$.

are measured with an electrostatic gridded energy analyzer. For the data presented in this paper, plasma flows are induced with a positively biased probe located at $R=41.2 \mathrm{~cm}$. Positive bias voltages induce flows with negative poloidal Mach numbers.

\section{EXPERIMENTAL RESULTS}

Fig. 4 shows the plasma radial current $I_{r}$ versus the poloidal Mach number for $P_{r f}=2.0 \mathrm{~kW}$ and $N_{n}=18$ (solid), 40 (dot) and 80 (dash) $\mu$ torr. The measured ion temperature and density at $P_{r f}=2.0 \mathrm{~kW}$ are $3.5 \mathrm{eV}$ and $2.0 \times 10^{11} \mathrm{~cm}^{-3}$, respectively $\left(v_{* i}=67\right)$. The poloidal Mach number is derived by normalizing the radial electric field at $R=41.3 \mathrm{~cm}$ with the product of the magnetic field strength $B_{0}$, ion thermal speed $v_{t}$, and the geometric factor $\varepsilon q^{-1}$. At $N_{n}=18 \mu$ torr, the plasma radial current curve consists of three different intervals: it increases sharply in the $-M_{p} \sim[2,15]$ interval, it decreases in the $[15,30]$ interval, and it increases in the $[30,110]$ interval. The slope of the radial current with respect to the poloidal Mach number is high, negative, and low in the first, second, and third interval, respectively. According to (1), the existence of both positive and negative slopes is an indication of the nonlinearity of the viscosity. The plasma radial current at $N_{n}=40 \mu$ torr consists of the same three intervals as well; however, the slope in the first and second intervals are smaller than those observed for the case when $N_{n}=18 \mu$ torr. At $N_{n}=80 \mu$ torr, however, the second interval (with negative slope) disappears and the amplitude of the radial current in the first interval becomes smaller. Measurements of ion temperature and line-average density in IMS show no dependence on neutral density. Therefore, according to (1), the reason for the difference in the magnitude and sign of the slopes for these curves is the change in neutral density.

Fig. 5 shows the same set of data for $P_{r f}=5.5 \mathrm{~kW}$. The measured ion temperature and density at $P_{r f}=5.5 \mathrm{~kW}$ are $7 \mathrm{eV}$ and $2.8 \times 10^{11} \mathrm{~cm}^{-3}$, respectively $\left(v_{* i}=28\right)$. The slope of the radial current curve for $N_{n}=18 \mu$ torr is high, negative (close to zero), and low in the $-M_{p} \sim$ 


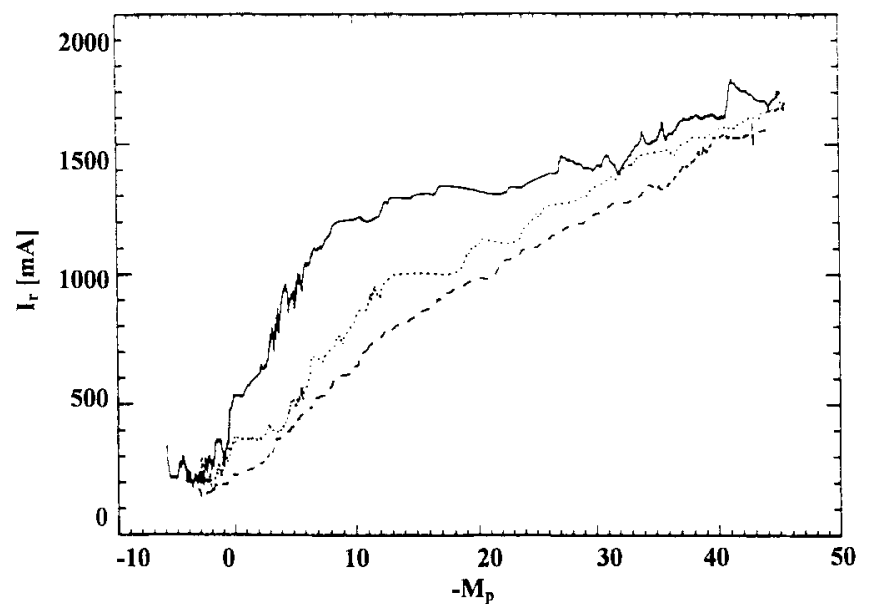

Fig. 5. The radial current versus Mach number for $N_{n}=18$ (solid), 40 (dotted), and 80 (dashed) $\mu$ torr, at $P_{r f}=5.5 \mathrm{~kW}$.

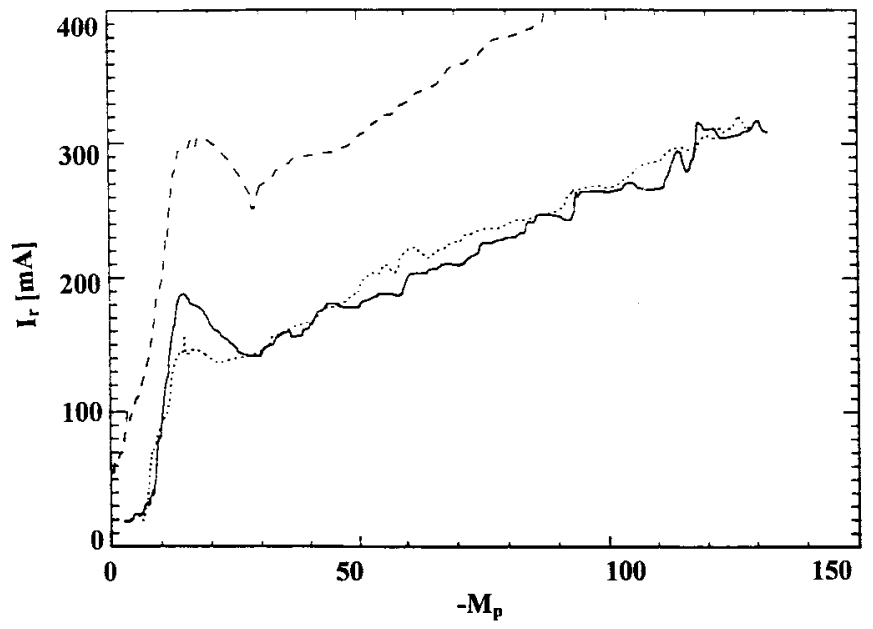

Fig. 6. The radial current versus Mach number for $N_{n}=40 \mu$ torr, $P_{r f}=0.5 \mathrm{~kW}$ (solid), $N_{n}=80 \mu$ torr, $P_{r f}=0.5 \mathrm{~kW}$ (dotted), and $N_{n}=40 \mu$ torr, $P_{r f}=1.0 \mathrm{~kW}$ (dashed).

$[-2,14],[14,26]$, and $[26,53]$ intervals. The radial current for $N_{n}=40 \mu$ torr consists of similar intervals. The slope of the curve for $N_{n}=80 \mu$ torr is nearly constant for all poloidal Mach numbers. Accordingly, an increase in neutral density diminishes the interval of zero or negative slope. Also, the drop in the radial current is diminished with an increase in input power.

Fig. 6 depicts the plasma radial current versus poloidal Mach number for $N_{n}=40 \mu$ torr, $P_{r f}=0.5$ (solid) and 1.0 (dash) kW, and $N_{n}=80 \mu$ torr, $P_{r f}=0.5 \mathrm{~kW}$ (dot). The measured ion temperature and density at $P_{r f}=0.5(1.0)$ $\mathrm{kW}$ are $1.8(2) \mathrm{eV}$ and $1.0(1.5) \times 10^{11} \mathrm{~cm}^{-3}$, respectively $\left[v_{* i}=168(112)\right]$. Lower ion temperatures and higher radial electric fields are the reasons for the wider range of poloidal Mach number, as compared to the other cases. Similar to Figs. 4 and 5, all of the curves consist of three distinct intervals: [5, 20], [20, 30], and [30, 150]. Intervals of negative slope do not exist at higher input powers for $N_{n}=80 \mu$ torr. The relative jump in the plasma radial current diminishes at higher input powers for $N_{n}=40 \mu$ torr.

\section{SUMMARY AND CONCLUSIONS}

A numerical code has been developed to study the effects of the plasma parameters on viscosity in a tokamak and a stellarator geometry using an expression developed by Shaing. The numerical calculations indicate that:

1) the poloidal viscosity in IMS has two local maxima, the toroidal peak at $M_{p} \sim 2$ and the helical peak at $M_{p} \sim 10$ (at $R=41.3 \mathrm{~cm}$ );

2) ion pressure and temperature gradient can break down the odd symmetry of the viscosity with respect to the poloidal Mach number and increase (decrease) the absolute value of viscosity for negative (positive) poloidal Mach numbers;

3) an increase in the plasma collisionality results in a decrease in the amplitude of the toroidal peak (in both tokamaks and classical stellarators) and an increase in the magnitude of the helical peak for the IMS case.

4) the local maximum of the total damping due to the nonlinearity of the parallel viscosity can be diminished by an increase in the neutral density.

With respect to the prediction 1): a drop in the bias current due to the helical peak is observed. A more accurate determination of the radial electric field based on the measurement of plasma potential profile at $P_{r f}=5.5 \mathrm{~kW}$ (not shown here) indicates that the peak of viscosity due to the helical ripple is at $M_{p} \sim-12 \pm 2$ in reasonable agreement with the numerical calculation [11]. No drop in the radial current due to the toroidal peak in the viscosity has been observed. The numerical calculations, however, predict two separate drops of the radial current due to the toroidal and the helical peaks. One of the implicit assumptions of Shaing's model is that the ion temperature and pressure gradients do not depend on the poloidal Mach number. The experimental results, however, show that the density gradient is dependent on the poloidal Mach number. A modification of Shaing's model, which includes the possibility of the dependence of the ion pressure and temperature gradients on the poloidal Mach number, may be required to explain the discrepancy between the theory and the experimental results for this case.

With respect to prediction 2): in another set of experiments, a preionization probe $\left(\mathrm{LaB}_{6}\right)$ was used to inject electrons into plasma and create an electric field with the opposite sign as that induced with the bias probe. Bias voltages and currents up to $-220 \mathrm{~V}$ and $-900 \mathrm{~mA}$ were applied and radial electric fields in $-5 \leq E_{r} \leq 0 \mathrm{kV} / \mathrm{m}$ range were measured. The input power and the neutral density were varied in $2-5$ $\mathrm{kW}$ range and 18-40 $\mu$ torr ranges. No drop in the radial current or jump in the radial electric fields was observed. The measured ion pressure and temperature gradients in the IMS were $V_{p p} \sim 4$ and $V_{p t} \sim 2$ for $P_{r f}=5.5 \mathrm{~kW}$. These results support the hypothesis that nonlinearity of viscosity in IMS diminishes for positive poloidal Mach numbers (possibly due to high ion pressure and temperature gradients at the plasma edge in IMS). 
According to the definition of the poloidal Mach number positive (negative) poloidal Mach numbers correspond to biasing the plasma edge with negative (positive) voltages. The experiments in continuous current tokamak [5] and tokamak experiment for technology oriented research [6] have shown that the radial current at which $\mathrm{H}$ mode is achieved with positive biasing is higher than that with negative biasing. Our numerical calculations suggest a possible explanation for this observation, namely, that this asymmetry may be caused by the effect of ion pressure and temperature gradient on viscosity at the plasma edge.

With respect to prediction 3): a decrease in the input power (from $5.5-0.5 \mathrm{~kW}$ ) results in a net increase in the plasma collisionality $\left(v_{* i}\right)$. The ion-neutral collision cross section increases with a decrease in ion temperature in the 1-7 eV range [15]. According to (1), the fact that the nonlinearity of viscosity in IMS can be observed more clearly at lower input powers is consistent with the hypothesis that the amplitude of the helical peak increases with an increase in plasma collisionality.

With respect to prediction 4): the experimental results presented in this paper indicate that the bifurcation of the radial electric field due to the nonlinear viscosity can be suppressed by an increase in the neutral density.

The study of parallel viscosity is important to understand how to improve the energy confinement time in toroidal magnetic devices such as tokamaks and stellarators. The work presented in this paper suggests that control of the plasma parameters and profile gradients, as well as the neutral density, is a possible means by which to modify the amplitude of the local maxima of the viscosity.

\section{ACKNOWLEDGMENT}

The authors would like to thank D. T. Anderson, F. S. B. Anderson, P. G. Matthews, and M. Coronado for their comments and valuable discussions. H. Dahi would like to thank P. Nonn for his technical support and assistance in maintaining the ECH source.

\section{REFERENCES}

[1] K. C. Shaing, "Test of tokamak low-mode-high-mode transition theory in stellarators," Phys. Fluids B, vol. 5, pp. 3841-3843, Nov. 1993.

[2] F. Wagner, G. Becker, K. Behringer, D. Campbell, A. Eberhagen, W. Engelhardt, G. Fussmann, O. Gehre, J. Gernhardt, G. V. Gierke, G. Haas, M. Huang, F. Karger, M. Keilhacker, O. Kluber, M. Kornherr, K. Lackner, G. Lisitano, G. G. Lister, H. M. Mayer, D. Meisel, E. R. Muller, H. Murmann, H. Niedermeyer, W. Poschenrieder, H. Rapp, H. Rohr, F. Schneider, G. Siller, E. Speth, A. Stabler, K. H. Steuer, G. Venus, O. Vollmer, and Z. Yu, "Regime of improved confinement and high beta in neutral-beam-heated divertor discharges of the ASDEX Tokamak," Phys. Rev. Lett., vol. 49, pp. 1408-1411, Nov. 1982.

[3] R. J. Groebner, "An emerging understanding of H-mode discharges in tokamaks," Phys. Fluids B, vol. 5, pp. 2343-2354, July 1993.

[4] K. C. Shaing and E. C. Crume, Jr., "Bifurcation theory of poloidal rotation in Tokamaks: A model for the L-H transition," Phys. Rev. Lett., vol. 63, pp. 2369-2372, Nov. 1989.

[5] R. J. Taylor et al., "H-mode behavior induced by cross-field currents in a Tokamak," Phys. Rev. Lett., vol. 63, pp. 2365-2368, Nov. 1989.

[6] R. R. Weynants and G. Van Oost, "Edge biasing in Tokamaks," Plasma Phys. Control. Nucl. Fusion Res., vol. 1, p. 473, 1990.
[7] G. L. Askinazi, V. E. Golant, S. V. Lebedev, V. A. Rozhanskij, and M. Tendler, "Radial current in a Tokamak caused by a biased electrode," Nucl. Fusion, vol. 32, no. 2, pp. 271-277, 1992.

[8] V. Erckmann, F. Wagner, J. Baldzuhn, R. Brakel, R. Burhenn, U. Gasparino, P. Grigull, H. J. Hartfuss, J. V. Hofmann, R. Jaenicke, H. Niedermeyer, W. Ohlendorf, A. Rudyj, A. Weller, S. D. Bogdanov, B. Bomba, A. A. Borschegovsky, G. Cattanei, A. Dodhy, D. Dorst, A. Elsner, M. Endler, T. Geist, L. Giannone, H. Hacker, O. Heinrich, G. Herre, D. Hildebrandt, V. I Hiznyak, V. I. Il'in, W. Kasparek, F. Karger, M. Kick, S. Kubo, A. N. Kuftin, V. I. Kurbatov, A. Lazaros, S. A. Malygin, V. I. Malygin, K. McCormick, G. A. Muller, V. B. Orlov, P. Pech, H. Ringler, I. N. Rio, F. Sardei, S. Sattler, F. Schneider, U. Schneider, P. G. Schuller, G. Siller, U. Stroth, M. Tutter, E. Unger, H. Wolff, E. Wurching, and S. Zopfel, "H mode of the W7-AS stellarator," Phys. Rev. Lett., vol. 70, pp. 2086-2089, Apr. 1993.

[9] K. Toi et al., "H-mode transition in the CHS heliotron/torsatron," Plasma Phys. Control. Fusion, vol. 36, pp. A117-122, July 1994.

[10] M. G. Shats, D. L. Rudakov, B. D. Blackwell, G. G. Borg, R. L. Dewar, S. M. Hamberger, J. Howard, and L. E. Sharp, "Improved particle confinement mode in the H-1 heliac plasma," Phys. Rev. Lett., vol. 77, pp. 4190-4193, Nov. 1996.

[11] H. Dahi, J. N. Talmadge, and J. L. Shohet, "Nonlinear viscosity in the interchangeable module stellarator," Physical Rev. Lett., vol. 77, pp. 3976-3939, May 1998.

[12] R. P. Doerner, D. T. Anderson, F. S. B. Anderson, P. H. Probert, J. L. Shohet, and J. N. Talmadge, "The magnetic field structure in a modular stellarator," Phys. Fluids, vol. 29, pp. 3807-3812, Nov. 1986.

[13] W. D. D'haeseleer, W. N. G. Hitchon, J. D. Callen, and J. L. Shohet, Flux Coordinates and Magnetic Field Structure. Berlin, Germany: Springer-Verlag, 1991.

[14] M. Coronado and J. N. Talmadge, "Evolution of the plasma rotation and the radial electric field for a toroidal plasma in the Pfirsch-Schlüter and plateau regimes subject to a biased electrode," Phys. Fluids B, vol. 5, pp. 1200-1212, Apr. 1993.

[15] C. F. Barnett et al., "Atomic data for controlled fusion research," Oak Ridge National Laboratory, Oak Ridge, TN, Rep. ORNL-5206 (vol. I),-5207 (vol. II), 1977.

Hossein Dahi received the B.S. degree in electrical and electronics engineering from Technical University of Budapest, Hungary, in 1989 and the Ph.D. degree in electrical and computer engineering from University of WisconsinMadison in 1996.

$\mathrm{He}$ is presently an Associate Research Scientist in the Department of Applied Physics and Applied Mathematics at Columbia University, NY. His research interests are in plasma physics and controlled fusion, including spectroscopic diagnostics and probes, transport and flow measurements; optics, including VUV and IR excimer light sources and lasers; and plasma aided manufacturing, including VHF reactive ion etching reactors.

Joseph N. Talmadge (M'83) received the B.S. degree in applied physics from Columbia University, New York, in 1974 and the Ph.D. degree in physics from the University of Wisconsin-Madison in 1982.

$\mathrm{He}$ is presently an Associate Scientist in the Department of Electrical Engineering at the University of Wisconsin-Madison where he is working on the Helically Symmetric Experiment, a new quasi-helically symmetric stellarator. His research interests are in plasma physics and controlled fusion and include plasma flow and electric field measurements in three-dimensional plasmas, transport, microwave and spectroscopic diagnostics, electron cyclotron heating, and MHD equilibrium and stability. 
J. Leon Shohet (S'56-M'62-SM'72-F'78) received the Ph.D. degree in electrical engineering from Carnegie Mellon University, Pittsburgh, PA, in 1961.

He served on the faculty of John Hopkins University, Baltimore, MD, before joining the University of Wisconsin-Madison faculty in 1966 where he was appointed Professor of Electrical and Computer Engineering in 1971. Currently, he is the Director of the Torsatron/Stellarator Laboratory, University of Wisconsin-Madison, a major department of energy fusion research facility, and is the Founding Director of the University's NSF Engineering Research Center for plasma-aided manufacturing as well as the past Chairman of the Department of Electrical and Computer Engineering. $\mathrm{He}$ is the author of two textbooks on plasma science, over 130 journal articles, and more than 400 conference papers. He holds six patents. His research interests include plasma-aided manufacturing, fusion (especially waves), instabilities, heating, confinement and diagnostics, communications, magnetohydrodynamics, electromagnetic field theory, biophysics, quantum electronics, and lasers.

Dr. Shohet is a Fellow of the American Physical Society. He received the Frederick Emmons Terman Award of the American Society for Engineering Education, the Merit Award of the IEEE's Nuclear and Plasma Sciences Society, the IEEE Richard F. Shea Award, the IEEE Plasma Science Prize, the IEEE Centennial Medal, and the John Yarwood Memorial Medal from the British Vacuum Council. He founded the IEEE Transactions on Plasma SCIENCE in 1973. 